\title{
Germanica
}

\section{Le « retour » de Victor Klemperer}

Victor Klemperers « Rückkehr»

\section{Anne-Marie Corbin}

\section{CpenEdition}

Journals

Édition électronique

URL : http://journals.openedition.org/germanica/2468

DOI : 10.4000/germanica.2468

ISSN : 2107-0784

Éditeur

Université de Lille

\section{Édition imprimée}

Date de publication : 30 décembre 2000

Pagination : 193-203

ISBN : 9782913857094

ISSN : 0984-2632

Référence électronique

Anne-Marie Corbin, « Le « retour » de Victor Klemperer », Germanica [En ligne], 27 | 2000, mis en ligne le 01 avril 2014, consulté le 06 octobre 2020. URL : http://journals.openedition.org/germanica/2468 ; DOI : https://doi.org/10.4000/germanica.2468

Ce document a été généré automatiquement le 6 octobre 2020.

(c) Tous droits réservés 


\title{
Le « retour » de Victor Klemperer
}

\author{
Victor Klemperers « Rückkehr »
}

Anne-Marie Corbin

1 En 1945, tout en travaillant à la LTI, son analyse de la langue du III ${ }^{\mathrm{e}}$ Reich, Victor Klemperer continue à rédiger ses carnets ${ }^{1}$. Mais il ne désire pas les publier dans leur ensemble, car il craint de livrer au public des réflexions trop personnelles. Fort heureusement, ceci ne l'empêche pas de noter ses impressions au jour le jour, comme il l'a toujours fait. Pour l'après-guerre, nous ne disposons encore que des notes des six premiers mois, la suite est annoncée à l'automne 1998.

2 L'éditeur présente cette œuvre comme une sorte de Volksbuch qui reflète l'humanisme de Klemperer et sa lutte contre les persistances du nazisme ${ }^{2}$. Effectivement, ces carnets nous livrent une mine de renseignements sur l'atmosphère qui régnait en Allemagne immédiatement après la victoire remportée sur le fascisme. Comment réagissaient les Allemands? Si le ravitaillement demeurait leur préoccupation essentielle, quelles étaient les autres? Quelles mesures furent prises dès les premiers jours pour que la vie recommence peu à peu ? A combien s'élevait le nombre des survivants qui, comme Klemperer, regagnaient leur ville et leurs maisons? Quel accueil leur réservaient ceux qui s'étaient tus lors des persécutions et des massacres perpétrés par les nazis? Les carnets de Klemperer répondent à toutes ces questions.

3 Nous nous limiterons cependant à trois aspects essentiels de ses confidences, qui correspondent à trois facettes de sa personnalité : le Juif qui assume sa judéité ; le communiste et la surprenante adhésion d'un homme plutôt attiré par la droite nationaliste avant 1933; enfin, l'universitaire et ses réflexions sur la politique culturelle et la formation dans la zone soviétique.

\section{Être Juif}

Dresde en juin 1945. Pour Klemperer, c'est un véritable paradis dont il serait le « centre triomphant » après les années d'humiliations qu'il a vécues (TA, 10). Il se donne pour objectif que les injustices commises envers les Juifs soient « réparées » immédiatement 
et dans leur totalité. Cependant, il ne voudrait pas apparaître aux yeux des Allemands comme " un triomphateur juif à l'esprit vengeur " ${ }^{3}$, flatté par ceux qui l'ont méprisé pendant des années $(\mathrm{TA}, 66)$. Il commence donc par témoigner de la reconnaissance à ceux qui l'ont aidé à l'époque nazie, et rassemble des signatures en faveur d'un entrepreneur qui a « racheté » des Juifs pour les sauver d'Auschwitz (TA, 27). Ou bien encore, il signale à la bienveillance de l'occupant soviétique le nom de celui qui, au péril de sa vie, a transporté en Angleterre des objets précieux, appartenant à des Juifs, pour les y mettre à l'abri (TA, 29). De telles démarches pouvaient, en effet, contribuer à sauver une entreprise, menacée de confiscation comme toutes celles dont les propriétaires s'étaient enrichis en exploitant la main d'œuvre étrangère ou juive. Par les mêmes ordonnances 124 et 126 des 30 et 31 octobre 1945, l'administration militaire (SMAD) prévoyait également la mise sous séquestre et la confiscation de toutes les propriétés de l'État allemand.

Dans l'immédiat, Klemperer se réjouit de disposer à nouveau d'une machine à écrire et d'avoir obtenu la carte de rationnement réservée normalement aux travailleurs de force, mais qu'on délivre aussi aux intellectuels. Sa femme peut également y prétendre en reconnaissance des souffrances qu'elle a endurées à ses côtés sous le III ${ }^{\mathrm{e}}$ Reich (TA, 15). En règle générale, Klemperer constate que les Juifs bénéficient d'un approvisionnement privilégié, une des premières mesures que prend l'occupant pour réparer le passé (TA, 119). Parce qu'il est juif et en raison de son grand âge, Klemperer obtient aussi une carte de priorité (TA, 54). Elle lui permettra d'échapper aux longues files d'attente où l'on se voit délivrer l'indispensable attestation de logement sans laquelle il est interdit de résider dans une ville allemande (TA, 96).

De retour à Dresde, sa femme et lui ont l'impression d'être les seuls rescapés (TA, 19) du monde juif qui fut englouti dans la tourmente (TA, 20) : «Ici, à Dresde, il y avait avant 1933 une communauté juive dont les membres se comptaient par milliers, elle est éliminée, il ne sont aujourd'hui même plus une centaine $»^{4}$. Un seul ami est revenu d'Auschwitz (TA, 79). De fait, les statistiques montrent que sur les 6000 Juifs de Dresde, il en restait 12 en 1945 ; des 1100 Juifs qui vivaient à Erfurt, 15 survécurent ; à Zwickau 5 seulement sur $460^{5}$. Comme dans bien des villes allemandes, le cimetière juif a été détruit (TA, 33), mais une synagogue vient de réouvrir ses portes à Berlin, donnant l'espoir que la vie puisse reprendre au grand jour (TA, 62). Klemperer n'ose cependant croire en un avenir meilleur: dans son entourage, les Allemands se refusent trop souvent à admettre toute l'envergure des exactions commises par les nazis envers les Juifs (TA, 37); en Saxe, il soupçonne même l'administration de ne leur être guère favorable (TA, 151). C'est pourquoi il redoute une nouvelle vague d'antisémitisme qui pourrait être plus grave encore qu'à l'époque nazie ${ }^{6}$. Ses craintes se fondent sur le sentiment que la population ressent la défaite de l'Allemagne comme la plus grande victoire jamais remportée par les Juifs, une victoire qu'on leur fera $"$ payer $»^{7}$. Cette hostilité, larvée pour le moment, serait d'autant plus dangereuse que les rares survivants juifs détiennent les postes clés, d'où l'envie qu'ils suscitent: «Parmi ces rares personnes, combien sont à des postes de direction $?^{8}$ »

7 Pour calmer ses inquiétudes, Klemperer tente d'assurer son propre avenir en faisant reconnaître les préjudices subis dans ce " jardin du paradis semé de mauvaises herbes » qu'il souhaiterait couper à la racine ${ }^{9}$. Et ce n'est pas chose facile, car les accords de Potsdam ne prévoient pas de dédommagement des Juifs (TA, 80). Un voisin des Klemperer réagit très violemment au fait que la confiscation des avoirs bancaires des 
particuliers touche aussi les Juifs. Afin que cesse cette injustice, il envisage de faire pression sur les autorités, en unissant ses forces à celles des autres Juifs concernés (TA, 110).

8 Autre problème: Klemperer se rend compte qu'il lui faudrait obtenir le statut de «victime du fascisme » $(\mathrm{TA}, 149)$ pour bénéficier d'un minimum de privilèges. Pour pallier les privations de l'époque, les « victimes du fascisme » ont droit, par exemple, à l'attribution de vêtements (TA, 204), à des suppléments de rations alimentaires, comme le fameux «Stollen » de Noël, et à une réduction d'impôts substantielle (TA, 210). Or, seules peuvent prétendre à ce titre les personnes incarcérées dans des camps de concentration pour des raisons politiques (TA, 149). Klemperer a raison d'être vigilant, car les Juifs ne furent jamais dédommagés en RDA. En outre, contrairement à ce qu'avait annoncé la SMAD en juillet 1946, elle ne restitua pas à leur propriétaires primitifs les biens confisqués sous le $\mathrm{III}^{\mathrm{e}}$ Reich et intégrés dans les entreprises nationalisées, les VEB (Volkseigene Betriebe).

Déjà la Deutsche Volkszeitung du 3 juillet 1945 donnait une interprétation très restrictive de ce terme: "Ils ont tous subi des préjudices graves et ils en ont souffert, mais ils n'ont pas tous combattu $»^{10}$. Cette définition provoqua un tollé chez tous ceux que les nazis avaient poursuivis pour des motifs raciaux ou religieux, dont les Juifs. En septembre 1945, les communistes de la zone soviétique durent lâcher du lest et admettre la reconnaissance des Juifs déportés comme "victimes du fascisme ». La distinction avec les «combattants du fascisme»- de fait, une hiérarchisation des souffrances endurées sous le $\mathrm{III}^{\mathrm{e}}$ Reich - avait des conséquences pécuniaires non négligeables sur le montant des retraites. Elle perdura jusqu'à la fin de la RDA, où les victimes reconnues du fascisme pouvaient partir à la retraite cinq ans plus tôt que la normale et obtenaient des facilités de séjours dans des maisons de repos ainsi qu'une pension (Ehrenpension) de 1350 marks par mois ${ }^{11}$.

Dans un tel contexte, il n'est donc pas étonnant que Klemperer soit envoyé d'un service à l'autre, qu'on l'adresse à l'assistance sociale et même à un Judenhilfswerk encore inexistant. Après avoir analysé les causes des difficultés qu'il rencontre pour faire reconnaître ses droits, Klemperer se rend compte du peu de valeur des trois certificats en sa possession, attestant que les nazis l'ont contraint à accomplir des travaux pénibles comme charrier des pierres dans une carrière. Avant 1933, il ne s'était pas engagé politiquement, ce dont on risque de lui tenir rigueur maintenant (TA, 181). C'est pourquoi il a conscience qu'il lui faut être membre de l'un des quatre partis du bloc démocratique, le KPD, le SPD, le LDPD ou la CDU (TA, 214).

\section{Devenir communiste}

11 Klemperer ne se sent pas d'affinités avec les Soviétiques, même si, en Saxe, les Allemands les accueillent avec la même animosité qu'ils témoignent aux Juifs - «Les Russes sont de retour, les Juifs aussi $»^{12}$. Ce n'est donc pas par solidarité avec les «Russes », que Klemperer va adhérer au Parti communiste. Leurs actes de pillage le révoltent (TA, 15). Il déplore le démontage des usines qui prennent le chemin de la Pologne ou de l'Union soviétique (TA, 36). Si le bétail n'est pas tué sur place (TA, 35), il est transporté en Union soviétique, ce qui ne manque pas d'accentuer encore la famine qui sévit en Allemagne (TA, 63). 
12 Malgré tout ce qu'il leur reproche et en dépit de leur «impérialisme» (TA, 176), Klemperer défend les Soviétiques contre la haine dont ils sont l'objet à Dresde (TA, 122). Une raison à cela : seule leur présence garantit, à ses yeux, qu'une guerre civile n'éclate en Allemagne (TA, 212). Contrairement à son entourage, Klemperer ne fait guère de différence entre les Alliés. Il met leur volonté d'anéantir l'industrie allemande sur le même plan. Au lieu de détruire sur place les machines - «nos» machines - les Soviétiques les transplantent chez eux. Les Alliés estiment tous que l'Allemagne doit expier $(\mathrm{TA}, 84)$ et le chômage n'est qu'une conséquence logique de la politique impitoyable menée par des vainqueurs, se comportant en terrain conquis (TA, 69). C'est pourquoi Klemperer ne se berce pas d'illusions sur les miracles que les Américains réaliseraient à Dresde s'ils venaient s'y installer en lieu et place des Soviétiques, comme l'espère une grande partie de la population (TA, 71). Mais cette perspective contribue à le faire réfléchir et à renforcer ses hésitations quand il s'agit de bien peser à quel parti politique adhérer.

13 Logiquement, la balance pencherait plutôt vers le KPD si les Soviétiques ne lui semblaient pas manifester si peu de sympathie pour les revendications des Juifs survivants (TA, 60). Klemperer retrouve une attitude similaire chez les membres du KPD qui se défient des Juifs, ne pouvant s'empêcher de faire l'amalgame entre eux et les capitalistes: «À l'évidence, elle discerne avec méfiance en tout Juif le commerçant, celui qui n'est pas ouvrier, le capitaliste $»^{13}$. En conséquence, si les dirigeants du KPD ont le choix, ils feront passer les intérêts des leurs avant ceux des Juifs. Klemperer reproche aussi aux communistes de s'engager trop peu pour l'université (TA, 60) alors qu'il faudrait y créer de nombreux postes de toute urgence (TA, 63). Ce philologue souligne fréquemment les analogies de leur langue avec celle des nazis (TA, 55).

Malgré tous ces aspects négatifs du KPD, Klemperer va peu à peu sortir de la défensive et laisser de côté ses hésitations (TA, 72). En effet, il éprouve de l'admiration pour certains fonctionnaires du KPD. Il décèle une grande sensibilité sous l'apparente dureté de l'un d'eux (TA, 99), dans un parti qui rassemble des braves gens et surtout des hommes de conviction (TA, 20). Sans ironie aucune, Klemperer souligne même l'idéalisme d'un fonctionnaire communiste, une forme de compliment qui n'aurait pu que déplaire à un marxiste (TA, 77). Cependant, il les trouve bien démunis face aux responsabilités qui leur incombent $(\mathrm{TA}, 20)$ et ne peut s'empêcher de les regarder avec condescendance et d'adopter un ton protecteur quand il assiste à leur démêlés avec l'administration (TA, 122). Malgré leur souci d'objectivité, sont-ils en mesure de faire la part entre les ordres de l'administration militaire et les exigences du terrain ? (TA, 172) Leur optimisme de commande n'est-il pas un réel handicap et comment le surmonter? (TA, 178).

Vite, Klemperer rentre dans un nouveau rôle d'homme de confiance (Vertrauensmann) des communistes, sans pour autant rompre avec les partis bourgeois (TA, 122). Il vise à devenir un médiateur qui aidera les "petits bourgeois ", pour qui les communistes sont des criminels, à dépasser ces préjugés (TA, 132). Il connaît bien le mépris et l'hostilité de la bourgeoisie envers ceux qui n'ont pas d'instruction, mais il ne les partage pas (TA, 43). Il désire également devenir le conseiller des communistes, l'homme capable de leur expliquer comment surmonter les difficultés qui se présentent, en mettant à leur disposition toute son expérience de la société bourgeoise : "Vous êtes trop tendres! Faire patte de velours ne vous permettra d'attirer aucun opposant du camp bourgeois » ${ }^{14}$. Le reproche de manifester trop de laxisme revient souvent sous sa plume, qu'il 
s'agisse de la réforme de l'agriculture ou de la fermeture des banques (TA, 145). Plus grave encore est l'arbitraire qu'ils font régner ${ }^{15}$. Klemperer estime que le KPD gagnerait en efficacité s'il comptait davantage d'intellectuels dans ses rangs (TA, 71), une condition déterminante à la fois pour le sort des intellectuels eux-mêmes - ce qui est évident - mais aussi pour l'avenir du parti, ce qui est plus douteux. On peut se demander si Klemperer ne surestime pas le rôle des intellectuels à l'intérieur des partis communistes et même dans l'histoire de l'Allemagne. Ou bien est-ce une manière de se persuader inconsciemment de sa propre importance?

Parfois Klemperer en vient presque au cynisme, quand il se targue de calculer froidement où réside son intérêt et de réagir en conséquence. Ce qui compte c'est « $m a$ situation, ma liberté ", il veut « miser sur le bon cheval ${ }^{16}$. Il enchaîne en disant que le KPD doit devenir une force qui permette au «travailleur intellectuel d'exercer ses droits $»^{17}$. Entre cette déclaration du 26 juillet et le 23 novembre 1945, moment où Klemperer se décide à remplir les formulaires pour adhérer au KPD, il s'écoule donc quatre mois d'hésitations. Le devenir de ses proches ne le laisse pas indifférent. Le professeur Wachs n'a pas obtenu de chaire parce qu'il était au SPD (TA, 154). Un autre collègue, un certain König, se demande s'il va entrer au centre ou à l'Union chrétienne (TA, 155), et Klemperer est tenté de suivre l'exemple d'un troisième qui rejoint le camp des libéraux (TA, 160). Peut-être l'attitude d'un catholique qui a choisi les communistes est-elle déterminante pour sa décision (TA, 183). Mais il se rend compte surtout que toute hésitation supplémentaire lui serait néfaste. S'il a encore l'impression de jouer la comédie (TA, 186), il va «annoncer la couleur $»^{18}$, sans prendre la peine d'assurer ses arrières en retardant, par mesure de sécurité, l'adhésion de sa femme au KPD (TA, 191), une mesure de prévention contre ce qui pourrait tourner mal ${ }^{19}$. Comme son mari, la femme de Klemperer estime que le KPD représente à la fois un moindre mal et une «nécessité » absolue ${ }^{20}$.

17 À la fin du formulaire qu'il vient de remplir, il s'explique sur ses motivations. Tout d'abord, le fait de ne pas être affilié à un parti serait devenu un luxe et presque de la lâcheté. Ensuite, seul un virage à gauche permettrait de sortir définitivement le pays de la misère et d'empêcher le retour des forces de la réaction qui ont amené les nazis au pouvoir. Le seul rempart contre tous ces maux serait devenu le KPD. Klemperer se sent tenu à ces explications pour deux raisons. Tout d'abord, il ne veut pas passer pour un simple opportuniste. Lui, le démocrate doit justifier un revirement qu'on pourrait lui reprocher (TA, 72). Il est bien conscient d'entamer une partie difficile où il joue l'équilibriste (TA, 77). En second lieu, il lui faut faire la preuve de la sincérité de cet engagement quelque peu tardif. A ces fins, il évoque l'irrésistible mouvement qui le pousse vers le KPD et le contraint à dépasser son apolitisme de jadis. Le dernier argument, c'est que son adhésion sera très profitable aux communistes, car cet exemple fera école (TA, 184).

La décision d'adhérer au parti devient pour cet intellectuel un impératif qui engage toute sa personne: «Il faut rentrer au KPD $»^{21}$. Cependant, il ne faut pas oublier que Klemperer rêve d'un KPD qui fonctionnerait comme un parti du centre, avec une aile droite et une aile gauche (TA, 77) et qui parviendrait à réaliser l'unité de la nation (TA, 72). Lui-même se situerait à gauche (TA, 85). Il évoque l'éventualité prochaine d'une fusion entre SPD et KPD à laquelle certains des communistes convaincus de son entourage ne croyaient pas encore fin 1945 (TA, 203). 
19 Autre raison majeure de l'adhésion de Klemperer au KPD : tout ceci est nécessaire pour « déblayer » le terrain, faire le ménage. Dans ce contexte, il peut paraître contradictoire que Klemperer délivre nombre de «Persilscheine », les certificats de blanchiment dont ont besoin les anciens membres du parti nazi pour se dédouaner face aux Alliés (TA, 12, $65,118,137,139,193,216)$. Il le fait souvent pour des personnes peu convaincues, coupables seulement d'avoir pactisé avec le diable pour conserver leur poste (TA, 116, 172). En revanche, il importe à Klemperer d'être impitoyable envers les juges nazis, aussi responsables, selon lui, que les SS et la Gestapo (TA, 38, 116). Mais il déplore le schématisme et la brutalité des purges qui risquent de semer la révolte dans une population qui avait, en majorité, adhéré au nazisme ${ }^{22}$.

\section{Répandre la culture}

20 Malgré les motifs avancés par Klemperer pour justifier son adhésion au KPD, on ne peut écarter l'hypothèse d'une stratégie de dernier recours pour obtenir enfin une chaire à l'Université alors qu'il voyait jusque là tous ses espoirs menacés (TA, 160). Et d'ailleurs, il ne dissimule pas cette arrière-pensée quand il se confie à ses carnets : «Je poursuis égoïstement l'arrière-pensée d'obtenir une chaire à l'Université $»^{23}$. Romaniste, il vise Leipzig ou Dresde (TA, 23) et n'attend pas que Friedrich Wolf revienne de Moscou en tant ministre de l'Éducation de la Saxe pour formuler cette demande (TA, 24). Un point positif en faveur de Klemperer : il n'a guère de concurrents, étant donné l'exode massif des universitaires à l'Ouest (TA, 61). Mais les choses ne se règlent pas aussi simplement qu'il l'espère. Tout d'abord, dans la zone soviétique, les priorités ne vont pas à la création d'universités, mais à celle de «Volkshochschulen» (TA, 161), c'est-à-dire d'écoles supérieures populaires, ou d'universités techniques. Une réforme - un moindre mal selon le commentaire de Klemperer $(\mathrm{TA}, 56)$ - touche l'enseignement général où s'impose la volonté d'unité dans la nouvelle école polytechnique de dix classes. Les cours y sont restructurés (TA, 82) pour mieux soumettre la formation au contrôle «scientifique » du marxisme (TA, 103).

21 Klemperer obtient satisfaction en deux temps. On reconnaît ses compétences en lui redonnant son titre d'Ordinarius le 19 octobre, ce qui l'autorise à percevoir un salaire. Le début des cours est fixé au mois de novembre (TA, 163). Après son adhésion au parti - en décembre 1945 - il obtient sa chaire à l'Université technique de Dresde (TA, 208) où un professeur fait office d'ouvrier de "troisième ordre ${ }^{24}$. Il sait que ses étudiants seront moins nombreux qu'auparavant : à Iéna, seuls 1100 sur 3000 sont autorisés à poursuivre leurs études; en priorité les «victimes du fascisme » ou leurs enfants, puis ceux qui ont collaboré à la reconstruction et les grands blessés de guerre (TA, 178).

Avec les communistes, Klemperer estime que la culture peut contribuer à la démocratisation de l'Allemagne si elle s'appuie sur «l'héritage culturel» pour promouvoir des classiques comme Lessing au théâtre. Il se réjouit qu'on célèbre l'anniversaire de Pestalozzi (TA, 207). Et Klemperer rêve, dans ce nouveau contexte, de présenter un jour Antigone à des ouvriers (TA, 207). Selon lui, les Soviétiques s'investissent davantage que les Américains dans la politique culturelle, car il leur faut lutter contre leur réputation de barbares ${ }^{25}$. Cela deviendra pour eux un atout supplémentaire, comme la création de la Ligue culturelle (Kulturbund) et le retour de J.R. Becher, célébré par les communistes (TA, 111). Klemperer adhère aux options de la Ligue, à laquelle il reconnaît, d'ores et déjà, la capacité d'acquérir l'autorité nécessaire 
pour infléchir les consciences vers la démocratie (TA, 113). Il participe aux activités de cette Ligue (TA, 194, 201), y faisant des conférences sur les « liens entre l'esprit et la matière » $(\mathrm{TA}, 120)$ et devenant le candidat unique des quatre partis représentés (TA, 198).

Si Klemperer défend la tolérance religieuse (TA, 215), s'il s'élève contre la propagande, diffusée à outrance sur les ondes (TA, 41, 78, 167, 172, 175), et réprouve la censure (TA, 143), il recommande de prendre des mesures sévères à l'encontre des professeurs qu'il soupçonne de vouloir «saboter " le nouveau régime, comme ils le firent à l'époque de la République de Weimar. Bien conscient que le KPD est fort mal perçu dans le corps professoral, Klemperer sent que la vigilance s'impose (TA, 191). En une formule lapidaire, il dénie aux universitaires la «liberté de trahir» un État dont ils n'approuvent pas les objectifs, et de détourner à ces fins leur traditionnelle liberté d'enseignement (TA, 201) : «La liberté de l'enseignement, ce n'est pas celle de saboter l'État $»^{26}$.

24 Ainsi, Klemperer réussit à reprendre une carrière, arrêtée sous le $\mathrm{III}^{\mathrm{e}}$ Reich, et à la poursuivre bien au-delà de l'âge de la retraite. Il est nommé à l'Université de Greifswald en 1947. À partir de 1950, il donne des cours à l'Université Humboldt de Berlin où il obtient une chaire pendant l'été 1951. L'année où Lion Feuchtwanger reçoit le Prix national de première classe, celui de troisième classe est décerné à Klemperer.

Au moment de la création de la RDA, Klemperer se montre quelque peu sceptique face à l'unanimité ambiante. Il lui semble revivre l'enthousiasme des foules pour Hitler. Une bonne moitié de la population est toujours aussi antisémite. Il craint encore que les antagonismes ne dégénèrent en guerre civile. Mais en 1951, les expériences qu'il a faites dans l'Allemagne d'Adenauer, où on le traite de "bolchevique de salon", le conduisent à approuver les positions de la RDA face aux « saboteurs » et aux « agents de l'ennemi de classe ", et il se lance dans des diatribes contre les Américains, justifiant ses prises de position contre l'Ouest par le fait que «la peste nazie a même imprégné la langue ».

26 À l'Université, il tente, cependant, de préserver la romanistique des rigueurs du marxisme. Il se sent investi d'une mission : préserver la langue allemande de toutes les dissonances qui mettraient définitivement en danger l'unité d'une nation que la Guerre froide soumet déjà à rude épreuve ${ }^{27}$. Sa dernière manifestation de soutien à une romanistique mise sous tutelle est une lettre non publiée adressée à la revue Sonntag le 3 juillet 1957 et dans laquelle il met les intellectuels en garde contre le dogmatisme et l'intolérance ${ }^{28}$.

Mais, pour son propre compte, Klemperer ne fait plus que des déclarations d'allégeance au régime. Lors des procès intentés aux médecins juifs à Moscou en 1953, il ne témoigne d'aucune compréhension pour la fuite des cerveaux de la communauté juive à l'Ouest. Sans distinguer la moindre trace d'antisémitisme dans cette nouvelle répression menée contre les Juifs, il ne veut y voir qu'une lutte de l'Union soviétique contre le sionisme dont il souligne les liens avec les États-Unis. Même le rapport Khrouchtchev, qui promettait une libéralisation du régime, semble une erreur à Klemperer qui n'élèvera pas non plus la voix pour défendre les intellectuels solidaires de la Révolution hongroise lors de l'affaire Harich/Janka. Le démocrate, l'observateur attentif, l'esprit indépendant, s'était volontairement coulé dans le moule du stalinisme. Il ne s'en dégagera plus. 


\section{NOTES}

1. . Victor Klemperer, Und so ist alles schwankend. Tagebücher Juni bis Dezember 1945 [désormais TA dans notre texte avec mention de la page citée], Berlin, Aufbau, 1996.

2. . Interview in Wochenpost, $n^{\circ} 39,26$ septembre 1996, p. 18.

3. . Victor Klemperer, Und so ist alles schwankend, op. cit., p. 15. «Ich mag nur nicht als jüdischer Rachegeist und Triumphator erscheinen ».

4. . Ibid., p. 195. «Hier in Dresden hat es vor 33 eine Judengemeinde von vierstelliger Zahl gegeben, sie ist ausgerottet, es werden heute keine 100 Juden mehr hier leben ».

5. . Lothar Mertens, "Juden in der DDR. Eine schwindende Minderheit ", in Deutschland Archiv, $\mathrm{n}^{\circ} 11,1986$, p. 1192.

6. . Victor Klemperer, Und so ist alles schwankend, op. cit., p. 195. «Es wird eine antisemitische Welle kommen, noch größer als die erste ».

7. I Ibid., p. 137. «Irgendwann wird den Juden einmal die Rechnung dafür präsentiert; ich sehe einen neuen Hitlerismus kommen, ich fühle mich durchaus nicht in Sicherheit ».

8. . Ibid., p. 195. « Aber wieviel von diesen wenigen sitzen auf leitenden Posten?»

9. . Ibid., p. 59. « Es wächst ein bißchen allzuviel Unkraut im Paradiesgarten. Trotzdem, wir leben in ihm, und die Hoffnung ihn auszujäten besteht ja ».

10. . Dieter Vorsteher, "Opfer des Faschismus ", in Parteiauftrag : ein neues Deutschland, Munich, Koehler \& Amelang, 1997, p. 152.

11. . «Juden in Deutschland », op. cit., p. 1193.

12. . Victor Klemperer, Und so ist alles schwankend, op. cit., p. 64. «Die Russen sind wieder da, die Juden sind wieder da $»$.

13. . Ibid., p. 36. "Sie wittert im Juden offenbar mit Mißtrauen den Kaufmann, Nicht-Arbeiter, Kapitalisten ».

14. . Ibid., p. 88. « Ihr seid zu sanft! Mit Euren Sammettpfötchen gewinnt ihr keinen bürgerlichen Gegner, verprellt Euch aber die eigenen Ànhänger ».

15. . Ibid., p. 170. «Willkür, Macht und Unberechenbarkeit der KPD scheint Faktum ».

16. I Ibid., p. 71. «Ich will nicht rein idealistisch entscheiden, sondern kalt berechnend, was für meine Situation, meine Freiheit, mein noch zu leistendes Werk das Beste ist, und damit doch meiner idealen Aufgabe dienend, auf das richtige Pferd setzen ».

17. . Ibid., p. 72. «Es muß eine einheitliche Arbeiterpartei sein (mit rechtem und linkem Flügel wie das Centrum), in der auch der geistige Arbeiter sein Recht hat ».

18. . Ibid., p. 186. «Aber ich muß nun wohl Farbe bekennen. - Eva tendiert zum Eintritt, und ich bin eigentlich dafür entschieden. Aber es kommt mir wie eine Komödie vor : Genosse Kl. ! Wessen Genosse?».

19. . Ibid., p. 187. « Das kann mein schwarzes und mein weißes Los bedeuten ».

20. . Ibid., p.191. «E. für ihren Teil glaubt bestimmt, daß KPD heute kleinstes Übel und Notwendigkeit bedeute ».

21. I Ibid., p. 75. «Ich : man muß zur KPD. - Er : Sie nannten sich neulich Demokrat! - Ich : Ja, aber KPD tut not !».

22. . Ibid., p. 216. «Hierdurch und durch die schematische Brutalität des « Säuberns » fühle ich den Boden schwanken. Eines Tages haben wir Aufruhr nach außen und Bürgerkrieg im Innern ».

23. . Ibid., p. 213. « Mein egoistischer Hintergedanke ist immerfort das Univ. -Katheder ».

24. . Ibid., p. 167. «Der Profesor steht als Arbeiter tertio loco».

25. . Ibid., p. 45. «Der Amerikaner braucht sich nicht als Kulturträger aufzuweisen, während der Russe erst einen Ruf zu gewinnen habe ».

26. . Ibid., p. 203. « Lehrfreiheit sei nicht Freiheit, den Staat zu sabotieren ». 
27. . Allocution tenue à la Ligue culturelle en 1952 à Berlin. « Wie oft habe ich Intellektuelle davor gewarnt, ihre doppelte Belastung durch bürgerliche Herkunft und studierten Beruf vertuschen zu wollen, indem sie sich doppelt gesinnungstüchtig gebärden ».

28. . In Wochenpost, n³9, 26 septembre 1996, p. 19.

\section{RÉSUMÉS}

En 1945, tout en travaillant à la LTI, son analyse de la langue du III ${ }^{\mathrm{e}}$ Reich, Victor Klemperer continue à rédiger ses carnets, récemment parus sous le titre Und so ist alles schwankend. Mais, à l'époque, il ne désire pas les publier dans leur ensemble, car il craint de livrer au public des réflexions trop personnelles.

Nous traiterons dans cet article trois aspects essentiels de ses confidences, qui correspondent à trois facettes de sa personnalité : le Juif qui assume sa judéité ; le communiste et la surprenante adhésion d'un homme plutôt attiré par la droite nationaliste avant 1933 ; enfin, I'universitaire et ses réflexions sur la politique culturelle et la formation dans la zone soviétique.

Während er 1945 an der LTI arbeitet, setzt Klemperer seine Tagebücher fort, deren erste 6 Monate jetzt in dem Band So ist alles schwamkend erschienen sind. Er aber wünscht es zu der Zeit nicht, sie allesamt zu veröffentlichen, denn er schreckt davor zurück, dem Leser allzu persönliche Überlegungen anzuvertrauen.

Der vorliegende Artikel befaßt sich mit drei Hauptthemen dieser Tagebücher, die drei Facetten seiner Persönlichkeit entsprechen : Klemperer als Jude, der zu seinem Judesein steht ; Klemperer als Kommunist, obwohl er vor 1933 eher auf der Seite der Deutschnationalen stand ; Klemperer schließlich als Professor, der sich über die Kulturpolitik und die Ausbildung in der sowjetischen Besatzungszone äußert.

\section{AUTEUR}

\section{ANNE-MARIE CORBIN}

Université du Maine - Le Mans 